\title{
MS, exercise, and the potential for older adults
}

\author{
Sean Horton • Dany J. MacDonald • Karl Erickson
}

Received: 15 May 2009 / Accepted: 10 March 2010/Published online: 31 March 2010

(C) European Group for Research into Elderly and Physical Activity (EGREPA) 2010

\begin{abstract}
Multiple Sclerosis (MS) is an autoimmune disorder of the central nervous system. The average onset of the disease is 30 years of age, and it afflicts women more often than men (ratio of approximately $2: 1$ ). The symptoms of the disease include fatigue, motor weakness, heat sensitivity, reduced mobility, abnormal gait mechanics, and poor balance. These symptoms decrease cognitive and physical functional capacity of an individual and tend to result in sedentary lifestyle behaviors. A sedentary lifestyle among individuals with MS increases the risk of secondary diseases such as coronary heart disease and obesity, particularly as one ages. The effect of exercise in treating symptoms of MS has been under explored, perhaps due to the fact that exercise was thought to magnify MS-related fatigue and other symptoms. Recent research has challenged this notion, advocating exercise as an effective therapy for the management of MS, as well as maintaining overall fitness and improving quality-of-life measures. While the research shows clear benefits, the barriers to exercise participation among MS patients are significant. Recommendations for various forms of exercise are provided, along with strategies for overcoming barriers to participation.
\end{abstract}

Keywords Multiple sclerosis · Aging · Physical activity

S. Horton $(\bowtie)$

Department of Kinesiology, University of Windsor,

Windsor, ON, Canada

e-mail: hortons@uwindsor.ca

D. J. MacDonald $\cdot$ K. Erickson

School of Kinesiology and Health Studies, Queen's University,

Kingston, ON, Canada
Recent reports indicate that between 55,000 and 75,000 Canadians live with multiple sclerosis (MS) and that approximately 1,000 new cases are diagnosed each year [34]. In the USA, roughly 400,000 individuals are affected by MS [29] with this number increasing by approximately 10,000 new cases per year [47]. Across European countries, the prevalence rates of MS are in the vicinity of 80 per 100,000 [5]. These statistics demonstrate that the disease is a global problem that affects hundreds of thousands of people worldwide.

MS is an autoimmune disease of the central nervous system that is characterized by destruction of the myelin that covers cell fibers in the brain and spinal cord, which leads to slowing or cessation of communication between the brain and body. This demyelination is also accompanied by axonal loss. The effect of the demyelination process and axonal loss is partial or total loss of functional capacity. MS typically develops between the ages of 15 and 40 years, but detection often occurs later in life due to the difficulties associated with accurately diagnosing the disease [47]. MS often progresses slowly, thereby delaying identification.

There are often profound effects on the quality of life and activities of daily living for people with MS. While there are many symptoms associated with the disease, fatigue is consistently reported as the most prevalent and persistent $[2,20,47]$. Defined as the lack of physical or mental energy to accomplish daily tasks, fatigue affects almost all those afflicted with MS. Bakshi [2] reports that between $65 \%$ and $97 \%$ of individuals have significant fatigue and $15 \%$ to $40 \%$ consider fatigue their most restricting symptom. The impact of fatigue is significant since it limits an individual's ability to perform daily tasks, is associated with decreased physical and mental activity [20] and is the primary cause of unemployment for those suffering from MS in the USA [47]. Importantly, Motl et al. 
[29] have shown that individuals with MS who have higher levels of physical activity report lower levels of fatigue.

Another symptom often reported by those suffering from MS is muscle weakness. This weakness can be the direct result of the decreased ability of the CNS to activate muscles in MS or from detraining as a result of reduced physical activity participation due to the symptoms associated with MS [13]. Research by Bakshi [2] shows that, in a sample of 656 individuals with MS, 45\% identified weakness as a symptom that impedes their activities of daily living. Clinical investigations of muscle strength measurement also show that individuals with MS are often weaker than control counterparts. In a review of previous studies, Ponichtera-Mulcare [39] noted that individuals with MS have typically been reported as generating significantly less force than controls on both knee extension and flexion. Even when initial strength deficits are not present, individuals with MS often experience greater fatigue, limiting strength output over time [46].

Related to fatigue and muscle weakness, balance problems are reported as a restrictive factor with MS. Poor balance inhibits an individual's ability to perform daily tasks while increasing the risk of falls, which often result in injuries. Bakshi [2] reports that $50 \%$ of those afflicted with MS indicated that poor balance limited their ability to accomplish daily tasks. It is also been reported that those with MS are up to 3.4 times more likely to fall and incur fractures due to poor balance [54]. The combination of poor balance, muscle weakness, and fatigue has ramifications with respect to an individual's ability to participate in regular physical activity.

Since there is presently no cure for MS, the remainder of an individual's life will be affected by the disease and the severity of the symptoms will dictate how an individual will live and function with the illness. Shannon [47] states that life expectancy with MS is reduced by approximately 1015 years. Confavreux and Compston [10] estimate that the median time from disease onset to death for people diagnosed with MS is 31 years. Considering that MS is typically diagnosed between the ages 15-40 years, many individuals live well into their senior years. This suggests that managing symptoms and understanding how the disease progresses throughout the aging process is critical to maximizing quality-of-life considerations.

It is clear that MS will have an impact on an individual's health and quality-of-life. Since those with MS typically have lower rates of physical activity than non-diseased people [28], they are at risk of additional health-related problems above and beyond symptoms associated with MS, ultimately increasing one's susceptibility to secondary diseases such as heart disease and obesity. Thus, increasing people's capacity to manage primary symptoms of MS and reduce the occurrence of secondary diseases is of foremost concern.

\section{Physical effects of exercise with MS}

Based on the primary symptoms of fatigue, muscle weakness, and loss of balance associated with MS, and increased risk for secondary health problems, it is important to examine the effectiveness of exercise interventions that address these specific issues. Examinations of the effects of exercise on individuals with MS have tended to differentiate between interventions designed to target muscular strength, typically through resistance training, and those designed to target cardio-respiratory fitness, typically through aerobic or endurance-based training methods. The following sections will provide an overview of the current understanding of the effects of exercise for individuals with MS. For a more detailed description of specific interventions, including a summary table, see the excellent review by Dalgas, Stenager, and Ingemann-Hansen [11].

Muscular strength While relatively few studies have examined physical activity/exercise interventions targeting muscular strength, the available empirical evidence suggests that resistance training at moderate intensities appears to be well tolerated (i.e., without symptom worsening) and can increase muscular strength in people with MS [11]. For example, DeBolt and McCubbin [12] conducted a randomized controlled trial of home-based resistance training with 36 individuals with MS. Over a period of 8 weeks, participants in the exercise condition completed a periodized program of lower body resistance exercises three times per week, with excellent adherence rates $(95 \%$ of exercise sessions completed). At post-test, leg extensor power improved significantly in the exercise group, though balance and general mobility (as measured by a timed Up and Go from sitting task) were unaffected. The reported lack of improvement in overall physical functioning is noteworthy, given the demonstrated increases in specific muscular function. However, this may be an artifact of the use of a time-based measure for physical functioning. Ease of movement and related indices may be more relevant to actual functioning in daily life. In this vein, anecdotal reports from exercise participants in this study included mention of having an easier time getting in and out of cars and going up and down stairs, while four participants stopped using a cane for regular locomotion over the course of the intervention.

White and colleagues [55] also examined the effects of an 8-week lower body progressive resistance training program. Twice per week eight participants performed lower body resistance exercises in a supervised setting. Resistance was increased by $2-5 \%$ when subjects were able to perform 15 repetitions of an exercise at a given weight. Knee extension, plantar flexion, and stepping performance on a 3-min step test all significantly improved from pre- to 
post-intervention. Significant increases in training volume, as represented by individually sequenced increases in resistance, were found between weeks 1,4 , and 8 , demonstrating participants' increased capacity for strengthbased activities as a result of the training protocol. Much like the anecdotal reports noted by DeBolt and McCubbin [12], such increases in capacity may well have important implications for activities of daily living. This is especially pertinent given that decreases in functional ability and the ability to perform activities of daily living are commonly associated with aging in general and particularly the progression of MS.

Taylor and colleagues [52] included upper body as well as lower body progressive resistance exercises in their training protocol. Performed twice weekly for 10 weeks, the nine participants increased leg strength, arm strength, leg endurance, and fast walking speed. The researchers also conducted qualitative interviews examining participants' experiences in the resistance training program [14]. In noting the overwhelmingly positive physical outcomes, participants mentioned perceptions of increased strength and endurance, decreased fatigue, and increased ability to perform activities of daily living. While difficult to measure objectively, the ability to perform activities of daily living such as walking up stairs and opening jars may be the most important physical outcomes of resistance exercise for people with MS. This may be particularly salient as these individuals grow older, given a common focus on such outcomes even in non-MS populations. This notion deserves further study, given the lack of research attention to exercise in aging populations with MS.

In considering the potential application of the findings from studies of resistance training and MS, a number of limitations must be recognized. Thus far, the effects of resistance training have been examined in studies with relatively small sample sizes [11], making concrete conclusions difficult. Of these studies, only three have been randomly controlled $[1,12,19]$ and the effects of differing intensities and resistance progressions have not been directly examined. These studies have only investigated resistance training for moderately impaired individuals $($ EDSS $<6.5)$. While logistics of resistance training will certainly present additional challenges for more severely impaired individuals, it is conceivable that effective (if somewhat limited) training protocols could be devised for individuals with EDSS scores of up to 8.0. Such individuals, while often restricted to a wheelchair, do retain some arm functioning which might be improved to aid in activities of daily living. Even for less-impaired individuals, little mention is made of specific modifications in exercise protocol necessary to account for neurologically based weakness common in MS, rather than simply detraining. If certain muscles (e.g., hip flexors, tibialis anterior) cannot be activated, some exercises may be impossible. Furthermore, the influence of age on the relationship between MS and resistance training is still unknown. While resistance training appears to benefit individuals with MS in a similar fashion to older adults experiencing similar mobility impairments, little is understood about how the process of aging with MS might affect tolerance of or response to various resistance training protocols.

Cardio-respiratory fitness Relative to resistance training and muscular strength, the effects of aerobic or endurance training on cardio-respiratory fitness have received much more research attention. Dalgas and colleagues [11] noted that endurance training at moderate intensities is well tolerated by individuals with MS. Furthermore, these individuals increased aerobic capacity ( $\mathrm{VO} 2 \mathrm{max}$ ) from training at least three times per week at that moderate intensity.

Specifically, Petajan and colleagues [37] conducted a randomly controlled trial with 54 individuals with MS to examine the effects of combined arm and leg ergometry exercise on cardio-respiratory fitness. Participants completed three 40 -min sessions per week at moderate $(60 \%$ of VO2 max) intensity for a 15 -week training period. At the completion of the training, participants in the exercise condition had significantly increased $\mathrm{VO} 2$ max and physical work capacity and significantly decreased skin fold thickness. Ponichtera-Mulcare et al. [40] conducted a similar study of aerobic exercise and obtained similar results. These researchers found that less-impaired participants (EDSS $<4.5$ ) showed the most improvement. This may suggest a relationship between training adaptation and level of neurological impairment; however, this finding has not been consistently replicated. Mostert and Kesselring [25] reported opposite trends, with more impaired participants improving most. While no consistent effects have been found in relation to functional capacity or activities of daily living [11], it would seem reasonable to suggest that tolerance of endurance training and the resulting increased capacity to exercise may well benefit individuals with MS in the prevention of secondary health problems such as heart disease and obesity.

Again, a number of limitations must be recognized within this body of literature. As with resistance training modalities, only moderately impaired individuals (EDSS < 6.5) working at moderate intensities have been studied. Thus, little is known about how well higher intensities are tolerated with regard to symptom worsening or how more severely impaired individuals respond to endurance training at any intensity. The effects of aging with MS on tolerance of endurance training are also unknown. It has been suggested that at moderate intensities, at least three training 
sessions per week are required to induce change in aerobic capacity $[11,48]$. It is unclear whether this is a tolerable workload for older and elderly individuals with MS. The general exercise literature suggests that older adults can derive considerable benefit from such a regimen (e.g., 15, 23), thus it is feasible that older adults with MS will benefit in a similar manner. As Elward and Larson [15] note, however, exercise programs are most effective when individualized to the specific objectives of the person or group in mind.

\section{MS, exercise, and quality-of-life}

The absence of a cure for MS makes quality-of-life (QOL) considerations paramount for both researchers and clinicians due to the fact that people must learn to live with and manage the disease [29]. An unfortunate consequence of living with $\mathrm{MS}$ is that patients score lower on QOL measures than control groups without MS and/or those suffering from other diseases [22]. For example, MS patients scored lower on QOL scales than individuals who had rheumatoid arthritis or inflammatory bowel disease [43]. The decreased functional capacity that tends to accompany the onset of MS is the main reason for the lower QOL scores reported by individuals with MS [11].

There has been a recent surge in research examining the effect of exercise on functionality, and a corresponding interest in the impact of exercise on QOL. A growing body of evidence suggests that substantial QOL benefits can be gained by MS patients who engage in exercise. Motl and Gosney's [27] meta-analysis of the impact of exercise on QOL determined an overall effect size of 0.23 , which the authors argue, compares favorably to the 0.3 effect size attributed to drug therapy. Their results highlight the recency of the research - of the 13 studies the authors cited, only one was conducted prior to 2000 [37], while the vast majority (10) of the studies were published in 2005 or later. Considering the positive nature of these initial findings, further work exploring the specific benefits of exercise on QOL appear worthwhile.

While Motl and Gosney [27] found an overall effect size of 0.23 , there were a number of noteworthy items within their findings that are worthy of elaboration. For example, the measurement tools that were used to measure QOL are an important consideration. Generally, studies that used a QOL tool specific to MS found that exercise had a beneficial effect on QOL whereas studies that used a more general measure of QOL were less likely to find measurable improvements. For instance, Schultz et al. [45] had participants engage in an 8-week aerobic bicycle training program. They looked at both a disease-specific QOL measure [Hamburg Quality of Life Questionnaire for Multiple Sclerosis (HAQUAMS)] along with generic scales [Medical Outcomes Study Short Forms (SF-36); Profile of Moods States (POMS)]. While HAQUAMS scores increased for the training group, none of the generic scales showed significant training effects. Similarly, Dalgas et al. [11] noted that studies measuring exercise-related changes in fatigue levels had varying results, but that this may simply reflect the instrument that was utilized to measure fatigue. This underscores the importance of employing a measurement tool that captures issues specific to the disease, for that will likely improve our understanding of the unique QOL benefits associated with exercise training for individuals with MS.

Motl and Gosney [27] reported a significant effect for aerobic exercise on QOL, but no significant effect for nonaerobic forms of exercise and no significant effect for studies that combined aerobic and non-aerobic exercise. For example, White et al. [55] found that strength training resulted in reduced self-reported fatigue levels in eight MS patients. Alternatively, Romberg et al. [42] randomly assigned 93 individuals to either an exercise condition (primarily resistance training) or to a control group. They found no effect on the Multiple Sclerosis Quality-of-Life scale, an MS-specific quality-of-life measure. The findings for non-aerobic forms of exercise are, therefore, somewhat mixed and certainly preliminary in nature. Motl and Gosney [27] acknowledged that it is premature to conclude that only aerobic exercise will improve QOL for those with MS. Due to the small number of studies looking at nonaerobic forms of exercise such as yoga or resistance training, this is a potentially fruitful area of future research.

Other factors appear to be related to the effect that an exercise program has on QOL measures. For participants who exercised for more than $90 \mathrm{~min}$ per week, the positive impact on QOL was larger $(d=0.44, \mathrm{CI}=.29-.59)$ than those exercising less than $90 \mathrm{~min}$ per week $(d=0.12$, $\mathrm{CI}=.01-.23)$. As might be expected, more exercise per week appears to translate into greater QOL benefits [27].

Of interest is that longer exercise programs, measured by duration in weeks, resulted in smaller QOL benefits than programs carried out over a shorter period of time. Motl and Gosney [27] examined programs of 3 months or longer and compared the findings to programs of less than 3 months. The longer programs resulted in modest improvements in QOL $(d=0.16, \mathrm{CI}=.07-.26)$ while shorter programs displayed greater QOL improvements $(d=0.34, \mathrm{CI}=.21-.47)$. The authors speculated that longer interventions may eventually lead to decreased enthusiasm by the participants as the workouts become tedious and less interesting. Making changes to a workout program may be an effective way of maintaining participant enthusiasm over time and thus maintaining QOL benefits. 
Furthermore, the social characteristics of the exercise program may influence its effectiveness and impact on QOL. For instance, Dodd and colleagues [14] qualitatively examined participants' experience in a resistance training program for individuals with MS. The participants highlighted the group atmosphere, developing new friendships, and the opportunity to work with other people with MS as key factors for their completion of the program. Research in the general exercise psychology literature has similarly demonstrated the significant role played by a group environment for the initiation, maintenance, and overall effectiveness of exercise [7]. Individuals exercising in groups have generally reported greater psychological and behavioral benefits than individuals exercising alone [8].

Participation trends In spite of the potential benefits to both physical functioning and QOL, people with MS generally display very low levels of physical activity involvement. In fact, MS patients score much lower on physical activity measures than the general population and are thus more prone to health complications that result from a sedentary lifestyle [33]. The reason for this may be partly due to entrenched attitudes towards exercise that have prevailed within the MS community. For many years, individuals with MS were advised to avoid exercise because of the concern over excessive fatigue and thermosensitivity $[11,38]$. A common consideration among those with MS was that exercise exertion would result in decreased energy for other activities. Fatigue is the most cited symptom by MS patients [2], and the widely held perception was that exercise would reduce energy stores. The emergence of research touting the benefits of exercise for managing the symptoms of the disease is relatively recent, but has led to its acceptance as a form of treatment [47]. Exercise participation rates, however, continue to be well below those of the general population and may continue in that vein until recent findings are widely disseminated.

Barriers to exercise participation associated with MS Even with more widespread acceptance of the benefits of exercise for individuals with MS, a number of individual-level barriers are known to influence exercise participation rates for this population. While some of these barriers are MS-specific, several are common to similarly impaired, older individuals. At the most basic level, MS subtype is known to affect exercise participation, with progressive types faring worse than relapsing types [31]. Related to subtype, symptom severity, frequency, and progression are constraints to exercise and physical activity $[26,32,50]$, perhaps as a reflection of functional disability $[31,33]$. In particular, increased fatigue is a serious impediment given the relatively high energy expenditure characteristic of exercise.
On a more general note, a lack of exercise self-efficacy, the belief in one's ability to successfully carry out the necessary physical activities, has been linked to decreased exercise participation $[24,30,50]$. Allied with a lack of self-efficacy are personal safety concerns [5], where an individual who is not confident in their exercise ability may feel at risk of injury, especially when exercise equipment is involved. Finally, lack of exercise enjoyment is also associated with less physical activity [30]. In spite of the established benefits of exercise for individuals with MS, these interrelated barriers must be overcome or addressed if exercise is to be a feasible option for this population, especially older individuals.

\section{Recommendations for exercise}

Resistance training Resistance training appears to be a well-tolerated method for improving muscular strength for individuals with MS. Though the response to such training in older populations has yet to be fully examined, key considerations should be observed. First, a focus of any resistance training program for individuals with MS should be lower body exercises as research has shown this to be the area of largest strength deficit in comparison to those without MS [46]. However, upper body exercises should be incorporated, as distinct gains are possible [52]. Training should begin at moderate intensities (i.e., $60-70 \%$ of one repetition maximum) with slow progression of resistance increases, as this is the only intensity empirically established as tolerable [11]. This may be increased contingent on individual tolerability as long as careful attention is given to symptom worsening. Exercises should be chosen to accommodate each individual's specific neurologically based weaknesses. These choices should target muscle groups the individual is actually able to activate and areas where strength improvements might aid in compensating for these weaknesses in activities of daily living. Schapiro [44] highlights that therapists or trainers "must carefully ferret out the muscles that can and should be strengthened by exercise" (p. 273). Finally, the salience of personal safety/environmental hazard concerns in a resistance training environment (i.e., perceptions of potentially dangerous, uncontrollable weights/machines) must be taken into account through education and appropriate supervision.

Aerobic training Given the susceptibility of MS populations, especially older individuals, to the secondary health risks associated with a relatively sedentary lifestyle, increasing physical activity and endurance-based aerobic exercise is of concern. This type of exercise appears to be well tolerated at moderate intensities (based on estimated $\mathrm{VO} 2$ max or predicted max heart rate), with three sessions 
per week necessary for fitness improvements [11]. While ergometer-type machines (lower body and upper/lower body combined) have been the most studied modality, treadmill [53] and aquatic $[18,51]$ training protocols have also demonstrated positive results. In particular, aquatic exercise allows for easier dissipation of body heat than land-based exercise, which may be of importance for thermosensitive individuals [54]. Thus, there may be room for creativity and variation in training modalities while still obtaining the desired fitness outcomes. This creativity and variation may play a key role in addressing issues of exercise enjoyment. Motl and Gosney's [27] meta-analysis on exercise and quality-of-life with MS included a comparison of exercise programs more than 3 months in duration with those 3 months or less. Participants involved in shorter programs reported greater QOL increases, and the authors speculated that continuing the same exercise protocol for a long period of time may lead to boredom and decreases in participant enthusiasm. By taking advantage of the different modalities available for aerobic exercise, it may be possible to prevent stagnation and maintain participants' enjoyment of the exercise process, although such an assertion warrants further investigation.

Other forms of exercise While the health benefits of resistance and endurance training are increasingly well established for individuals with MS, several alternative forms of exercise may also be beneficial. Oken and colleagues [36] conducted a randomized controlled intervention consisting of weekly yoga practice. Participants showed significant improvements in fatigue measures after 6 months compared with the control group. Similarly, Tai Chi and other balance and flexibility-based exercise modalities have been suggested as potentially fruitful for individuals with MS [38, 47]. Petajan and White [38] proposed pyramids of muscular fitness and physical activity, progressing from passive range of motion work and basic activities of daily living to integrated strength training programs and structured aerobic training programs. These pyramid progression strategies suggest that there are gains to be made at all activity levels and that more impaired individuals do not necessarily need to begin with a full training protocol if they are not able. This may be an especially important consideration for older individuals with MS who might otherwise avoid exercise entirely.

Regardless of the exercise modality chosen, there are a number of recommendations common to all. First, exercising individuals with MS are subject to considerations above and beyond healthy populations, particularly in relation to neurologically based patterns of muscle weakness and general fatigue. Many questions remain regarding a number of factors surrounding MS and exercise, notably the lack of specific guidelines for older individuals. Therefore, any exercise program should be overseen by qualified and knowledgeable professionals. Dodd and colleagues [14] noted the importance participants placed on having a knowledgeable and encouraging fitness leader and the benefits of a group exercise setting. Additionally, upon initiation of any program, exercisers with MS should be made aware of the possibility of some temporary symptom worsening, which generally recede with no overall deleterious effect [49]. Any exercise program should also be individualized, taking into account each exerciser's specific symptoms, degree of impairment, and exercise tolerance.

It is also of utmost importance to address known barriers to exercise participation for individuals with MS. Without concern for these barriers, participants may have difficulty adhering to even the most efficacious training protocol. Taking a cue from the exercise psychology and health promotion research fields, existing theoretical models of behavior change may be useful in designing appropriate strategies to overcome even MS-specific barriers to exercise participation. Brawley [6] highlighted both the prudence and practicality of such an approach for general exercise interventions. Brawley noted the effectiveness of designing interventions around "a few efficient predictors that reliably tell us something about behavior" (p. 112). Regarding MS specifically, the Theory of Planned Behaviour [17] contains variables related to outcome expectations, perceived social norms, and control beliefs, corresponding well with the barriers to physical activity and exercise outlined in previous MS research. In the same vein, Bandura's Social Cognitive Theory (SCT) [3, 4] with self-efficacy as a principle component, has been used as a guiding framework to examine correlates of current physical activity for individuals with MS [24, 30]. SCT's emphasis on selfefficacy might lend itself to intervention designs targeting efficacy-specific barriers to exercise participation. Given the salience of these psychological factors as barriers to exercise, more behavior change theory-driven MS intervention research and practice is warranted.

\section{Future directions}

Studies in the past few years point to the beneficial aspects of exercising with MS, and this growing body of evidence has changed the prevailing attitudes from avoidance of exercise to one of cautious enthusiasm. While there is no cure for MS, exercise appears to be beneficial at multiple levels, and it may have an important role to play in delaying negative symptoms of the disease. For example, animal research has suggested that exercise might provide an antiinflammatory effect, potentially slowing the disease process 
[21]. Extending this research to human participants is an important next step in MS research.

In addition, exercise has an important impact on QOL. A comparison to the therapeutic effects of drugs suggests that exercise-related improvements in QOL are large enough to be of clinical relevance [27]. While investigations of exercise and QOL in MS patients have multiplied in the last few years, this area of research is still in its early stages. For instance, conflicting evidence has been reported for the relationship between MS symptom severity and depression [e.g., 9, 16]. It remains to be seen how symptom severity affects overall QOL and how this relationship may be influenced by exercise.

The majority of studies have examined the effect of aerobic exercise on MS; there have been comparatively few examinations of non-aerobic forms of exercise such as yoga or resistance training on functional capacity and QOL of individuals with MS. Of those studies, many were conducted with a small number of participants, thus it is difficult to make definitive conclusions regarding their effects. Motl and Gosney's [27] meta-analysis found an effect on QOL for exercise programs that were aerobically based, but not for other forms of training (i.e., resistance exercise, combined training). The authors did acknowledge that the small number of studies made this a premature conclusion to draw. In particular, the differential benefits accrued from aerobic exercise targeting reduction in secondary health risks (i.e., heart disease, diabetes, etc.) versus resistance training directly targeting strength, mobility, and balance for activities of daily living are worth considering. Dalgas et al. [11] suggested that resistance training of moderate intensity appears to be well tolerated and improve both muscle strength and certain functional measures among moderately impaired MS patients. Considering that non-aerobic exercise may result in fewer fatigue-related symptoms, further exploration of alternative programs of conditioning would prove worthwhile.

Future studies may be well served by utilizing a QOL measure specific to MS. Studies that used MS-specific QOL measures were more likely to find improvements than instruments more generic in nature [33]. Generic measures may not capture the unique obstacles MS patients encounter with the disease.

The vast majority of studies to date have been conducted on individuals who have a relatively high level of functioning (EDSS levels of 6.5 or better), thus there is scant evidence as to how exercise will affect those more severely impaired. Individuals with an EDSS of higher than 7 have not been studied to any meaningful degree which points to another important avenue for future investigation.

There has been very little research on exercise specific to older individuals with MS. The slow deterioration in functional capacity that occurs with MS over time can make the initiation and maintenance of an exercise program particularly difficult for older individuals. It is important, therefore, to examine the effects of exercise on more highly impaired, older adults as they start exercise programs at comparatively later ages. It may be that some forms of exercise are more tolerable and/or enjoyable depending on severity of symptoms and the stage of the disease. In addition, longitudinal studies examining the effects of consistent exercise training over a number of years, and the potential for limiting symptom worsening as one ages, would prove especially valuable.

Dalgas et al. [11] recommended that future studies should evaluate longer training periods; indeed, the majority of studies have been cross-sectional in nature and relatively short in duration. The authors suggest that a program of at least 12 weeks is recommended in order to maximize physiological adaptations. Of interest is the fact that Motl and Gosney [27] found greater QOL improvements for programs that were fewer than 12 weeks than those that were conducted over longer periods of time. The challenge for both researchers and practitioners is to provide programs that are varied enough to keep participants interested and engaged so that they will derive the full benefits of long-term involvement. In addition to the specifics of the exercise program, social support, both within the exercise setting (i.e., exercise staff, other participants) and outside the exercise setting (family, friends) will affect participant enjoyment and adherence to exercise (e.g., 35, 41). Specific to MS, Motl et al. [30] reported that physical activity is indirectly associated with improved QOL by way of social support and self-efficacy for managing MS, although further research would be valuable to further explicate these relationships.

Finally, there has been very little in the way of qualitative inquiry into people's exercise experience with MS [5]. Given the individual nature of the disease and the important role that self-efficacy plays in exercise involvement [29], there is likely to be important information derived through qualitative discussions of participant experiences. In-depth qualitative interviews may provide insights that fixed-response questionnaires will fail to uncover. Specifically, discussions around exercise preferences, social support, and barriers to exercise involvement will prove valuable for understanding the unique circumstances of individuals and provide in-depth insights into factors that affect exercise participation and quality-of-life over the lifespan.

\section{References}

1. Aimeta M, Lampichlera J, Musila U, Spiesbergera R, Pelikana J, Schmida J et al (2006) High and moderate intensities in strength training in multiple sclerosis. Isokin Exerc Sci 14:153 
2. Bakshi R (2003) Fatigue associated with multiple sclerosis: diagnosis, impact, and management. Mult Scl 9:219-227

3. Bandura A (1986) Social foundations of thought and action: a social cognitive theory. Prentice Hall, Englewood Cliffs, NJ

4. Bandura A (1997) Self-efficacy: the exercise of control. W.H. Freeman and Company, New York

5. Borkoles E, Nicholls A, Bell K, Butterly R, Polman R (2008) The lived experiences of people diagnosed with multiple sclerosis in relation to exercise. Psych Health 23:427-441

6. Brawley LR (1993) The practicality of using social psychological theories for exercise and health research and intervention. J Appl Sport Psychol 5:99-115

7. Brustad RJ, Babkes ML (2004) Social influence on the psychological dimensions of adult physical activity involvement. In: Weiss MR (ed) Developmental sport and exercise psychology: a lifespan perspective. Fitness Information Technology, Morgantown WV, pp 313-332

8. Carron AV, Hausenblas HA, Mack D (1996) Social influence and exercise: a meta-analysis. J Sport Exercise Psy 18:1-16

9. Chwastiak L, Ehde DM, Gibbons LE, Sullivan M, Bowen JD, Kraft GH (2002) Depressive symptoms and severity of illness in multiple sclerosis: epidemiologic study of a large community sample. Am J Psychiatry 159:1862-1868

10. Confavreux C, Compston A (2006) The natural history of multiple sclerosis. In: Compston A (ed) McAlpine's multiple sclerosis, 4th edn. Elsevier, Philadelphia PA, pp 183-284

11. Dalgas U, Stenager E, Ingemann-Hansen T (2008) Multiple sclerosis and physical exercise: recommendations for the application of resistance-, endurance- and combined training. Mult Scl $14: 35-53$

12. DeBolt LS, McCubbin JA (2004) The effects of home-based resistance exercise on balance, power, and mobility in adults with multiple sclerosis. Arch Phys Med Rehabil 85:290-297

13. De Haan A, de Ruiter CJ, Van der Woude LHV, Jongen PJH (2000) Contractile properties and fatigue of quadriceps muscles in multiple sclerosis. Muscle Nerve 23:1534-1541

14. Dodd KJ, Taylor NF, Denisenko S, Prasad D (2006) A qualitative analysis of a progressive resistance exercise programme for people with multiple sclerosis. Disabil Rehabil 28:1127-1134

15. Elward K, Larson EB (2002) Benefits of exercise for older adults. A review of existing evidence and current recommendations for the general population. Clin Geriatr Med 8:35-50

16. Feinstein A, Feinstein K (2001) Depression associated with multiple sclerosis: looking beyond diagnosis to symptom expression. J Affect Disorders 66:193-198

17. Fishbein M, Ajzen I (1975) Belief, attitude, intention, and behavior: an introduction to theory and research. AddisonWesley, Reading, MA

18. Gehlsen GM, Grigsby SA, Vinant DM (1984) Effects of an aquatic fitness program on the muscular strength and endurance of patients with multiple sclerosis. Phys Ther 64:653-657

19. Harvey L, Smith A, Jones R (1999) The effect of weighted leg raises on quadriceps strength, EMG parameters and functional activities in persons with multiple sclerosis. Phys Ther 85:154-161

20. Krupp LB, Alvarez LA, LoRocca NG, Scheinberg LC (1988) Fatigue in multiple sclerosis. Arch Neurol 45:435-437

21. LePage C, Bourdoulous S, Beraud E, Couraud PO, Rieu M, Ferry A (1996) Effect of physical exercise on adoptive experimental auto-immune encephalomyelitis in rats. Eur J Appl Physiol 73:130-135

22. Lobentanz IS, Asenbaum S, Vass K, Sauter C, Klosch G, Kristoferitsch W et al (2004) Factors influencing quality of life in multiple sclerosis patients: disability, depressive mood, fatigue and sleep quality. Acta Neurol Scand 110:6-13

23. Mazzeo RS, Tanaka H (2001) Exercise prescription for the elderly: current recommendations. Sports Med 31:809-818
24. Morris KS, McAuley E, Motl RW (2008) Self-efficacy and environmental correlates of physical activity among older women and women with multiple sclerosis. Health Educ Res 23:744-752

25. Mostert S, Kesselring J (2002) Effects of a short-term exercise training program on aerobic fitness, fatigue, health perception and activity level of subjects with multiple sclerosis. Mult Scler 8:161-168

26. Motl RW, Arnett PA, Smith MM, Barwick FH, Ahlstrom B, Stover EJ (2008) Worsening of symptoms is associated with lower physical activity levels in individuals with multiple sclerosis. Mult Scler 14:140-142

27. Motl RW, Gosney JL (2008) Effect of exercise training on quality of life in multiple sclerosis: a meta-analysis. Mult Scler 14:129-135

28. Motl RW, McAuley E, Snook E (2005) Physical activity and multiple sclerosis: a meta-analysis. Mult Scler 11:459-463

29. Motl RW, McAuley E, Snook E, Gliottoni R (2009) Physical activity and quality of life in multiple sclerosis: intermediary roles of disability, fatigue, mood, pain, self-efficacy and social support. Psych Health Med 14:111-124

30. Motl RW, Snook E, McAuley E, Scott JA, Douglass ML (2006) Correlates of physical activity among individuals with multiple sclerosis. Ann Behav Med 32:154-161

31. Motl RW, Snook E, McAuley E, Scott JA, Hinkle ML (2007) Demographic correlates of physical activity in individuals with multiple sclerosis. Disabil Rehabil 29:1301-1304

32. Motl RW, Snook EM, Schapiro RT (2008) Symptoms and physical activity behavior in individuals with multiple sclerosis. Res Nurs Health 31:466-475

33. Motl RW, Snook EM, Wynn DR, Vollmer T (2008) Physical activity correlates with neurological impairment and disability in multiple sclerosis. J Nerv Ment Dis 196:492-495

34. MS Society of Canada (2009) Multiple sclerosis society of Canada. Available at http://www.mssociety.ca. Accessed 3 Apr 2009

35. Oka RK, King AC, Young DR (1995) Sources of social support as predictors of exercise adherence in women and men ages 50 to 65 years. Women Health 1:161-175

36. Oken BS, Kishiyama S, Zajdel D, Bourdette D, Carlsen J, Haas M et al (2004) Randomized controlled trial of yoga and exercise in multiple sclerosis. Neurology 62:2058-2064

37. Petajan J, Gappmaier E, White A, Spencer M, Mino L, Hicks R (1996) Impact of aerobic training on fitness and quality of life in multiple sclerosis. Ann Neurol 39:432-441

38. Petajan JH, White AT (1999) Recommendations for physical activity in patients with multiple sclerosis. Sports Med 27:179-191

39. Ponichtera-Mulcare JA (1993) Exercise and multiple sclerosis. Med Sci Sports Exerc 25:451-465

40. Ponichtera-Mulcare JA, Mathews T, Barret PJ, Gupta SC (1997) Change in aerobic fitness of patients with multiple sclerosis during a 6 month training program. Sports Med Train Rehabil 7:265-272

41. Resnick B, Orwig D, Magaziner J, Wynne C (2002) The effect of social support on exercise behavior in older adults. Clin Nurs Res 11:52-70

42. Romberg A, Virtanen A, Ruutiainen J (2005) Long-term exercise improves functional impairment but not quality of life in multiple sclerosis. J Neurol 252:839-845

43. Rudick RA, Miller D, Clough JD, Gragg LA, Farmer RG (1992) Quality of life in multiple sclerosis: comparison with inflammatory bowel disease and rheumatoid arthritis. Arch Neurol 49:1237-1242

44. Schapiro RT (2006) Managing the symptoms of multiple sclerosis. In: Cook SD (ed) Handbook of multiple sclerosis, 4th edn. Taylor \& Francis, New York, pp 271-280

45. Schulz K, Gold S, Witte J, Bartsch K, Lang U, Hellweg R et al (2004) Impact of aerobic training on immune-endocrine parame- 
ters, neurotrophic factors, quality of life and coordinative function in multiple sclerosis. J Neurol Sci 225:11-18

46. Schwid SR, Thornton CA, Pandya S, Manzur KL, Sanjak M, Petrie MD et al (1999) Quantitative assessment of motor fatigue and strength in MS. Neurology 53:743-750

47. Shannon JB (2007) Multiple sclerosis sourcebook. Omnigraphics Inc., Detroit

48. Slawta JN, McCubbin JA, Wilcox AR, Fox SD, Nalle DJ, Anderson G (2002) Coronary heart disease risk between active and inactive women with multiple sclerosis. Med Sci Sports Exerc 34:905-912

49. Smith RM, Adeney-Steel M, Fulcher G, Longley WA (2006) Symptom change with exercise is a temporary phenomenon for people with multiple sclerosis. Arch Phys Med Rehabil 87:723-727

50. Snook EM, Motl RW (2008) Physical activity behaviors in individuals with multiple sclerosis: roles of overall and specific symptoms, and self-efficacy. J Pain Symptom Manage 36:46-53
51. Sutherland G, Anderson MB, Stoové MA (2007) Can aerobic exercise training affect health-related quality of life for people with multiple sclerosis? J Sport Exerc Psych 23:122-135

52. Taylor NF, Dodd KJ, Prasad D, Denisenko S (2006) Progressive resistance exercise for people with multiple sclerosis. Disabil Rehabil 28:1119-1126

53. Van den Berg M, Dawes H, Wade DT, Newman M, Burridge J, Izadi H, Sackley CM (2006) Treadmill training for individuals with multiple sclerosis: a pilot randomized trial. J Neurol Neurosurg Psychiatry 77:531-533

54. White L, Dressendorfer R (2004) Exercise and multiple sclerosis. Sports Med 34:1077-1100

55. White LJ, McCoy SC, Castellano V, Guierrez G, Stevens JE, Walter GA, Vandenborne K (2004) Resistance training improves strength and functional capacity in persons with multiple sclerosis. Mult Scler 10:668-674 